\title{
The Structural Integrity of an Amygdala-Prefrontal Pathway Predicts Trait Anxiety
}

\author{
M. Justin Kim and Paul J. Whalen \\ Department of Psychological and Brain Sciences, Dartmouth College, Hanover, New Hampshire 03755
}

Here, we used diffusion tensor imaging (DTI) and showed that the strength of an axonal pathway identified between the amygdala and prefrontal cortex predicted individual differences in trait anxiety. A functional magnetic resonance imaging (fMRI) functional localizer that has been shown to produce reliable amygdala activation was collected in 20 psychiatrically healthy subjects. Voxelwise regression analyses using this fMRI amygdala reactivity as a regressor were performed on fractional anisotropy images derived from DTI. This analysis identified a white matter pathway between the amygdala and ventromedial prefrontal cortex. Individual differences in the structural integrity of this putative amygdala-prefrontal pathway were inversely correlated with trait anxiety levels (i.e., higher pathway strength predicted lower anxiety). More generally, this study illustrates a strategy for combining fMRI and DTI to identify individual differences in structural pathways that predict behavioral outcomes.

\section{Introduction}

Evidence from the nonhuman animal literature indicates that the ventromedial prefrontal cortex (vmPFC), including the medial orbitofrontal cortex and ventral anterior cingulate cortex, is reciprocally connected with the amygdala, both functionally and anatomically (Amaral et al., 1992; Milad and Quirk, 2002; Ghashghaei et al., 2007). In humans, functional magnetic resonance imaging (fMRI) studies have used functional connectivity methods to evaluate the relationship between the amygdala and vmPFC (Pezawas et al., 2005; Hare et al., 2008). These data will be bolstered by evidence in the human of actual structural connections between the amygdala and vmPFC, which can only be inferred from nonhuman animal data.

One method that holds promise for delineating such connections in the human brain is diffusion tensor imaging (DTI). This neuroimaging method measures the strength and direction of water diffusivity (i.e., anisotropy) in brain tissue. A popular scale that measures the degree of anisotropy is fractional anisotropy (FA), which is thought to be modulated by the degree of myelination, axonal membrane thickness and diameter, and/or amount of parallel organization of axons (Basser and Pierpaoli, 1996; Beaulieu, 2002). Thus, FA is interpreted as an indicator of white matter pathway strength, or integrity. Of critical relevance to the present study is a recent DTI study that demonstrated an axonal pathway between the amygdala and vmPFC (Johansen-Berg et al., 2008).

Here, we sought to combine fMRI and DTI by using individual differences in amygdala reactivity as the basis for identifying

Received May 18, 2009; revised July 21, 2009; accepted Aug. 10, 2009.

This work was supported by National Institute of Mental Health Grant MH080716. We thank Erika J. Ruberry, Alison L. Duffy, and Rebecca A. Loucks for technical assistance.

Correspondence should be addressed to M. Justin Kim, Department of Psychological and Brain Sciences, Dartmouth College, 6207 Moore Hall, Hanover, NH 03755. E-mail: justin.m.kim@dartmouth.edu. DOI:10.1523/JNEUROSCI.2335-09.2009

Copyright $\odot 2009$ Society for Neuroscience $\quad 0270-6474 / 09 / 2911614-05 \$ 15.00 / 0$ individual differences in the structural integrity of connections between the amygdala and prefrontal cortex. To this end, we had subjects view fearful versus neutral facial expressions, a task that has been shown to reliably activate the human amygdala. Individual differences in these activations across subjects were regressed against the strength of FA signal values focusing on potential connections with the prefrontal region. Given previous studies demonstrating lower anxiety levels in subjects showing a greater functional coupling between the amygdala and prefrontal cortex (Pezawas et al., 2005), we planned to regress individual differences in the strength of this pathway against the range of trait anxiety scores in our subject sample to determine whether the strength of amygdala-prefrontal structural connectivity inversely predicted individual differences in trait anxiety levels.

\section{Materials and Methods}

Subjects. Twenty healthy volunteers ( 12 women; $21.1 \pm 4.15$ years of age; 20 right-handed) were screened for current or past psychiatric illness (axis I or II) using the Structured Clinical Interview for DSM-IV (SCID). No subjects had ever taken psychotropic medications. The study protocol was approved by the Committee for the Protection of Human Subjects at Dartmouth College. Written, informed consent was obtained from the participants before the experiment.

Stimuli. Faces with fearful and neutral expressions from six different individuals (three males and three females) were used (Ekman and Friesen, 1976). The faces were normalized for size and luminance. All of the stimuli were backprojected onto a screen, on which the subjects viewed using a mirror that was mounted on the head coil.

Procedure. Subjects were asked to passively view fearful and neutral faces during scanning presented separately in alternating $18 \mathrm{~s}$ blocks, interleaved with $18 \mathrm{~s}$ blocks showing a single crosshair at the middle of the screen. Within each $18 \mathrm{~s}$ face block, a total of 36 fearful or neutral faces were presented on a black background. Each face was shown on the screen for $200 \mathrm{~ms}$ with a fixed interstimulus interval of $300 \mathrm{~ms}$, consistent with previous studies (Kim et al., 2003). The order of the faces within a block was pseudorandomized to ensure that the same face was not presented more than twice in a row. The order of face blocks was counterbalanced across subjects. Each scan was $4 \mathrm{~min}$ and $14 \mathrm{~s}$ long. Immediately 
after the scanning session, all subjects were asked to rate the valence (scale from 1 to 9: 1 , very negative; 3 , negative; 5 , neither negative nor positive; 7 , positive; 9 , very positive) and arousal (scale from 1 to 9: 1 , very low; 3 , low; 5 , medium; 7 , high; 9 , very high) levels of each face that they had seen in the scanner. Valence and arousal ratings from one subject were unavailable because of technical problems. After the scanning sessions, each subject's anxiety level was assessed with the State Trait Anxiety Inventory (STAI-S; STAI-T) (Spielberger et al., 1988) self-report questionnaires. In addition, the subjects completed the Beck Depression Inventory (BDI) (Beck et al., 1961). Handedness was determined with the Edinburgh Handedness Inventory (Oldfield, 1971).

Image acquisition. All subjects were scanned on a 3.0 tesla Philips Intera Achieva Scanner (Philips Medical Systems) equipped with a SENSE birdcage head coil. Anatomical T1-weighted images were collected using a high-resolution three-dimensional magnetization-prepared rapid gradient echo sequence, with 160 contiguous $1 \mathrm{~mm}$ thick sagittal slices [echo time (TE), $4.6 \mathrm{~ms}$; repetition time (TR), $9.8 \mathrm{~ms}$; field of view (FOV), 240 $\mathrm{mm}$; flip angle, $8^{\circ}$; voxel size, $1 \times 0.94 \times 0.94 \mathrm{~mm}$ ]. Functional images were acquired using echo-planar T2*-weighted imaging sequence. Each volume consisted of 36 interleaved $3 \mathrm{~mm}$ thick slices with $0.5 \mathrm{~mm}$ interslice gap (TE, $35 \mathrm{~ms}$; TR, $2000 \mathrm{~ms}$; FOV, $240 \mathrm{~mm}$; flip angle, 90; voxel size, $3 \times 3 \times 3.5 \mathrm{~mm}$ ). Diffusion weighted images were collected using echoplanar imaging with 70 contiguous 2 -mm-thick axial slices and 32 noncollinear diffusion gradients (TE, $91 \mathrm{~ms}$; TR, $9013 \mathrm{~ms} ; b$ value, 1000 $\mathrm{s} / \mathrm{mm}^{2}$; FOV , $240 \mathrm{~mm}$; flip angle, $90^{\circ}$; voxel size, $1.875 \times 1.875 \times 2 \mathrm{~mm}$ ).

Data analysis. Anatomical and functional images were processed using Statistical Parametric Mapping software (SPM2) (Wellcome Department of Imaging Neuroscience, London, UK). Amygdala blood oxygen level-dependent (BOLD) signal ( $\beta$ weights) from the significantly activated voxels for the fearful versus neutral contrast were extracted for each subject and used as a predictor variable in subsequent voxelwise regression analysis with FA images (for details, see supplemental Methods, available at www.jneurosci.org as supplemental material). Diffusionweighted images were preprocessed using the Diffusion Toolbox and FSL software of the Oxford Centre for Functional MRI of the Brain (Smith et al., 2004). Diffusion data were corrected for eddy current and head movement, and then the tensor model was fitted to calculate FA values for each voxel, producing one FA image per subject. To generate spatially normalized individual FA images, each individual image was aligned into Montreal Neurological Institute (MNI)-152 standard space using nonlinear registration (Rueckert et al., 1999). After spatial normalization, FA images were smoothed using a Gaussian kernel of $6 \mathrm{~mm}$ full width at half-maximum. Voxels with FA values $<0.2$ were removed before statistical analyses, and an additional white matter mask derived from the Wake Forest University Pick Atlas (Maldjian et al., 2003) was applied to the images to ensure the results were attributable to white matter.

To generate a statistical map in which local FA values were correlated with amygdala BOLD signals, preprocessed FA images were entered into a general linear model with amygdala BOLD signals from fearful versus neutral contrast as a covariate of interest using SPM2. Given our focus on fibers connecting the amygdala with the vmPFC [Johansen-Berg et al. (2008), their Fig. 4] (we will refer to this as the "amygdala-vmPFC pathway") and the lack of well defined anatomical region of interest mask for this region, a significance threshold of $p<0.05$ corrected for multiple comparisons over the frontal and temporal white matter tissue $(\sim 82,000$ $\mathrm{mm}^{3}$, which encompasses the spatial location of the amygdala-vmPFC pathway) was used, as determined by Monte Carlo simulations implemented in AlphaSim within AFNI software (Cox, 1996). For regions about which we had no a priori prediction, a more conservative significance threshold of $p<0.00001$ (uncorrected, $34 \mathrm{~mm}^{3}$ ) was used (Shin et al., 2005). FA values of the significant voxels were extracted from FA images that had been normalized and not smoothed to eliminate the effects of smoothing (Tuch et al., 2005). Extracted FA values from these voxels were used for additional statistical investigations, including correlation analyses with trait anxiety measures (supplemental Methods, available at www.jneurosci.org as supplemental material).

\section{Results}

\section{Behavioral data}

Mean valence ratings of fearful faces (mean $\pm \mathrm{SD}, 2.91 \pm 0.85$ ) were significantly more negative than neutral faces $(4.60 \pm 0.41$; $\left.t_{(18)}=-7.14 ; p<0.001\right)$. Fearful faces $(5.19 \pm 1.42)$ were also rated as significantly more arousing than neutral faces (3.38 \pm $\left.0.98 ; t_{(18)}=6.41 ; p<0.001\right)$. Descriptive statistics for self-report measures are as follows: STAI-S, $32.85 \pm 7.03$; STAI-T, $37.15 \pm$ 8.25; BDI, $3.40 \pm 3.97$. These results show that overall subjects perceived fearful faces as negative and arousing, and all scores for anxiety and depression were within the normal range.

\section{Amygdala reactivity to fearful versus neutral faces}

As expected, there were significant BOLD signal increases in the amygdala to fearful faces versus neutral faces. The most prominent signal increases were observed at the lateral border of the left ventral amygdala $\left(\mathrm{MNI}-33,3,-24 ; t_{(19)}=3.70 ; p<0.05\right.$ corrected).

\section{DTI voxelwise regression analysis}

Left ventral amygdala responses to fearful versus neutral faces were positively correlated with local FA values signaling the presence of white matter fibers located between the amygdala and the medial prefrontal cortex. Critically, these significantly correlated voxels were located within an amygdala-vmPFC pathway independently identified by Johansen-Berg et al. (2008) using tractography. Significantly correlated voxel clusters extended from the left dorsal amygdala/substantia innominata region (MNI - 27, 3, $\left.-11 ; t_{(18)}=2.14\right)$, through the left ventral striatum (MNI $-18,3$, $\left.-8 ; t_{(18)}=2.70\right)$, and terminated within the left medial orbitofrontal cortex $\left(\mathrm{MNI}-17,25,-20 ; t_{(18)}=4.51\right)$ (Fig. 1, Table 1).

The identification of this pathway was specific to its correlation with amygdala responses to fearful faces since a similar correlation was observed to fearful versus the fixation baseline $(r=0.559 ; p<0.05)$ but not to neutral versus fixation $(r=$ $-0.383 ; p=0.10)$. No voxels within the amygdala-vmPFC pathway were negatively correlated with amygdala BOLD responses to fearful versus neutral faces. Valence and arousal ratings of the faces did not show significant relationships with FA values. FA values, which range from 0 to 1.0 , were $>0.3$, mitigating concern that these effects were related to any gray matter differences as these are typically observed with FA values $<0.2$.

There was some evidence of correlation with white matter located within the right hemisphere, but this effect was confined to a right ventral striatum $\left(\mathrm{MNI} 25,6,-12 ; t_{(18)}=3.69\right)$ region similar to that on the left (Fig. 1, coronal slices, $Y=0-9$ ) and did not show evidence of consistent correlation throughout a pathway to the prefrontal cortex.

\section{Correlations with anxiety}

Individual differences in trait anxiety scores were negatively correlated with the mean $\mathrm{FA}$ value of the entirety of the identified amygdala-vmPFC pathway $(r=-0.515 ; p<0.05)$ (Fig. 2$)$. The magnitude of this effect was strongest in the posterior region $\left(\mathrm{MNI}-10,12,-11 ; t_{(18)}=4.12\right)$ of the amygdala-vmPFC pathway (supplemental Fig. 1, available at www.jneurosci.org as supplemental material).

To determine the anatomical specificity of this relationship between FA and trait anxiety, an additional whole-brain voxelwise regression analysis was performed on the FA images, using trait anxiety as a regressor. Results showed that the relationship between FA and trait anxiety is specific to white matter 


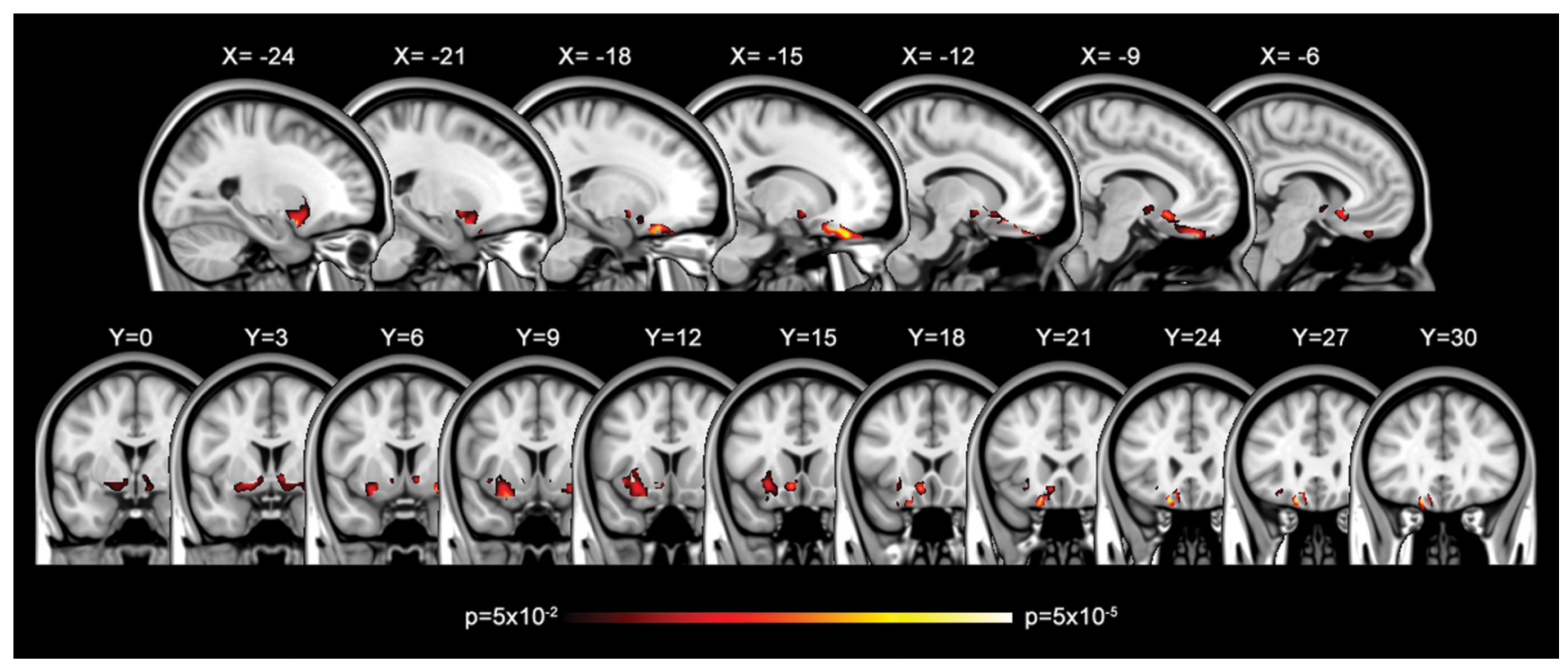

Figure 1. Amygdala-vmPFC pathway identified based on variability in functional activation of the amygdala. Statistical map showing FA voxels that are positively correlated with amygdala reactivity to fearful versus neutral faces ( $p<0.05$, corrected) in sagittal and coronal slices. The color bars indicate $p$ values.

Table 1. Nearest gray matter brain regions of voxels within an amygdala-vmPFC pathway that was identified based on the variability in functional activation of the amygdala

\begin{tabular}{llrrrr}
\hline \multirow{2}{*}{$\begin{array}{l}\text { Nearest gray matter } \\
\text { brain regions }\end{array}$} & $\begin{array}{l}\text { Cluster } \\
\text { size }\left(\mathrm{mm}^{3}\right)\end{array}$ & \multicolumn{3}{l}{ MNI coordinates } & \\
\cline { 3 - 5 } & \multicolumn{1}{c}{$x$} & $y$ & \multicolumn{1}{c}{$z$} & $t$ \\
\hline L orbitofrontal cortex & 5321 & -17 & 25 & -20 & 4.51 \\
L anterior cingulate cortex & & -8 & 16 & -10 & 3.48 \\
L subcallosal gyrus & & -24 & 8 & -17 & 3.83 \\
L putamen & & -18 & 3 & -8 & 2.70 \\
Lamygdala & & -27 & 3 & -11 & 2.14 \\
R putamen & 1426 & 25 & 6 & -12 & 3.69 \\
R caudate & & 10 & 5 & -4 & 2.62 \\
R pallidum & & 18 & 2 & -7 & 2.40 \\
\hline
\end{tabular}

L, Left; R, right.

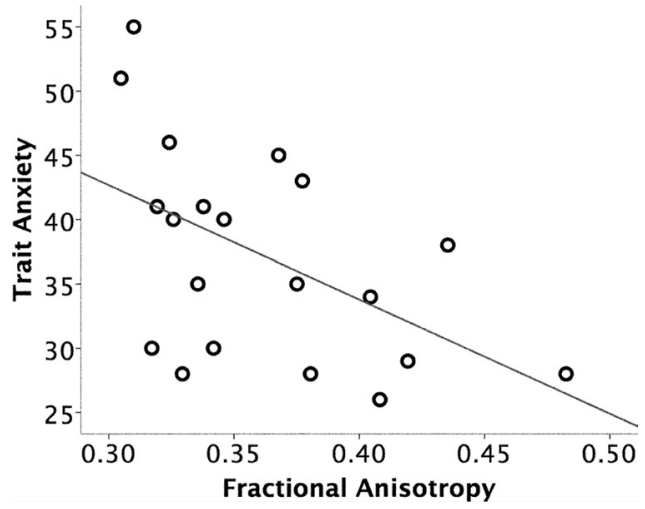

Figure 2. Correlation between the structural integrity of an amygdala-vmPFC pathway and trait anxiety. FA values of an amygdala-vmPFC pathway ( $r=-0.515 ; p<0.05)$, shown in Figure 1, were negatively correlated with trait anxiety.

fibers within the frontal and temporal areas, primarily overlapping with the originally defined amygdala-vmPFC pathway (supplemental Fig. 2, available at www.jneurosci.org as supplemental material).

Trait anxiety was not correlated with amygdala BOLD signal increases to fearful versus neutral faces $(r=-0.222 ; p=0.35)$.

\section{Discussion}

In this study, we used individual differences in fMRI responses in the amygdala to identify a white matter pathway between the amygdala and vmPFC using DTI. We then independently demonstrated that individual differences in the structural integrity of this pathway predicted differences in trait anxiety levels.

A compelling aspect of the present data set is that the FA correlation map-which simply shows the spatial locations of voxels in which FA values were associated with amygdala reactivity to fearful faces - was almost exclusively allocated within the probabilistic tractography results that identified a similar amygdala-vmPFC pathway in an independent study (Johansen-Berg et al., 2008). We observed white matter circuitry that connected the lateral ventral amygdala with the dorsal amygdala extending in a superior direction into the substantia innominata region of the ventral basal forebrain, ventral striatum/nucleus accumbens, and vmPFC. These data converge with a previous DTI study of both human and macaque brains that identified comparable patterns of anatomical connectivity across both species, including extensive connections between the amygdala and vmPFC (Croxson et al., 2005). Our data extend these findings by showing that individual differences in the strength of this pathway can be used to predict behavioral outcomes, in this case, trait anxiety.

We are careful to refer to the identified white matter as a "putative" pathway because there are a number of candidate white matter sources for the FA values observed here. First, the more lateral FA voxels could be consistent with the location of the uncinate fasciculus (UF). The UF is the major fiber tract connecting the anterior temporal lobe to the inferior frontal cortex (Ebeling and von Cramon, 1992; Highley et al., 2002). Critically, the UF interconnects the amygdala and the orbitofrontal lobe (Ebeling and von Cramon, 1992). In Figure 1, the most lateral FA voxels, just lateral and ventral to the putamen (see coronal slices, $Y=6-18$ ), are consistent with the location of the UF. But both the present study and the previous findings of Johansen-Berg et al. (2008) also reveal more medial white matter. These could potentially represent shorter, multisynaptic tracts between the amygdala and the ventral basal forebrain (Aggleton et al., 1980) and striatum (Fudge et al., 2002) and then connec- 
tions from these regions and the orbitofrontal cortex (Ferry et al., 2000). However, they could represent fiber tracts projecting in a lateral direction that serve to connect more medial gray matter structures (e.g., amygdala, nucleus basalis) with the UF [Selden et al. (1998), their Fig. 3C].

Since the anatomical connections between the amygdala and vmPFC are bidirectional (Amaral et al., 1992; Ghashghaei et al., 2007), the present data may suggest that more coherent structural connectivity between the amygdala and vmPFC, implying the possibility of more efficient communication and information exchange between the two brain regions, predicts more favorable outcomes in terms of anxiety. Indeed, a positive correlation between structural and functional connectivity between the amygdala and vmPFC has been demonstrated, such that individuals with stronger anatomical connections between these two brain regions demonstrate stronger functional connectivity (Wang et al., 2009). Furthermore, a recent study showed an inverse relationship between low-expressing serotonin transporter gene (5HTTLPR) alleles, which have been known to be associated with anxiety or depression, and FA values of the left frontal uncinate fasciculus, which overlaps with the amygdala-vmPFC pathway in our study (Pacheco et al., 2009). Together with the present results, these studies collectively suggest that the coherence of white matter between the amygdala and vmPFC is strongly linked with beneficial functional outcomes in terms of anxiety.

These structural results complement the functional findings of Pezawas et al. (2005), who showed that increased functional coupling between the amygdala and vmPFC predicted lower levels of temperamental anxiety. This study also reported that only the functional connectivity of the amygdala-vmPFC was associated with anxiety-whereas other measures, including amygdala reactivity to fearful faces, similar to our findings, were not. The lack of a strong relationship between amygdala reactivity to fearful faces and anxiety suggests that, in psychiatrically normal subjects, assessing the strength of the connectivity between the amygdala and vmPFC, be it structural or functional, could be a better predictor of anxiety.

That said, it should be noted that some studies have reported a significant positive correlation between amygdala reactivity and measures of anxiety (Bishop et al., 2004; Etkin et al., 2004; Haas et al., 2007; Dickie and Armony, 2008). Interestingly, we note that the majority of these findings have linked amygdala reactivity to unaware or unattended fearful faces and anxiety (Bishop et al., 2004; Etkin et al., 2004; Dickie and Armony, 2008), and thus, the present effect may be related to our use of overtly presented fearful faces.

In the present study, a positive correlation was observed between the strength of this amygdala-vmPFC pathway and amygdala reactivity to fearful faces. Consistent with this finding, Pezawas et al. (2005) demonstrated that amygdala activity was positively correlated with vmPFC activity. These findings might at first seem counterintuitive since numerous studies have shown an inverse relationship between the amygdala and vmPFC (Kim et al., 2003; Shin et al., 2005; Urry et al., 2006; Hare et al., 2008). First, these inverse effects have been observed during tasks in which subjects regulated their responses to presented stimuli. Thus, whether an inverse relationship is observed between the amygdala and the vmPFC may depend heavily on the nature of the task used. Perhaps the current task using passive viewing of fearful faces was the basis of the observed identified pathway and future DTI studies could use paradigms that call for more active regulation for comparison. Second, other studies showing an inverse relationship between the amygdala and vmPFC involved clinically pathological groups (Rauch et al., 2000; Shin et al., 2005; Etkin and Wager, 2007). For example, Shin et al. (2005) observed such an effect during the passive viewing of fearful faces in a group of subjects with posttraumatic stress disorder. Thus, perhaps the exaggerated amygdala responses observed in patient groups call for attempts by the vmPFC to regulate such responses (Shin et al., 2005; Etkin and Wager, 2007). DTI studies of these patient populations combined with these behavioral tests during fMRI could help clarify these issues.

Finally, we note that there are numerous constructs that can be used to measure the propensity for negative affect, or negative temperament. For example, although conceptually similar, trait anxiety as measured by the STAI is not identical with neuroticism (Costa and McCrae, 1997) nor harm avoidance (Cloninger, 1986). Harm avoidance is defined as a tendency to respond intensely to aversive stimuli and as an aversion to punishment, novelty, and nonreward (Cloninger, 1986), whereas neuroticism is a tendency to experience negative emotions and interpret situations as threatening (Costa and McCrae, 1997). To date, brain imaging studies of prefrontal-amygdala interactions have used only one of these available measures within a given study [e.g., STAI (Etkin et al., 2004; present study); harm avoidance (Pezawas et al., 2005); neuroticism (Haas et al., 2007)]. Future studies using multiple measures will be needed to dissect the relationship between these related but ultimately unique measures of negative affect and prefrontal-amygdala connectivity.

In conclusion, our data provide evidence that the strength of an anatomical amygdala-prefrontal pathway predicted lower levels of normal trait anxiety. Given that one of the easiest and most convenient ways to elicit human amygdala activation is to overtly present fearful faces, our data offer a simple strategy for combining fMRI and DTI to identify structural pathways that can then predict behavioral outcomes.

\section{References}

Aggleton JP, Burton MJ, Passingham RE (1980) Cortical and subcortical afferents to the amygdala of the rhesus monkey (Macaca mulatta). Brain Res 190:347-368.

Amaral DG, Price JL, Pitkanen A, Carmichael ST (1992) Anatomical organization of the primate amygdaloid complex. In: The amygdala: neurobiological aspects of emotion, memory, and mental dysfunction (Aggleton JP, ed). New York: Wiley.

Basser PJ, Pierpaoli C (1996) Microstructural and physiological features of tissues elucidated by quantitative-diffusion-tensor MRI. J Magn Reson B 111:209-219.

Beaulieu C (2002) The basis of anisotropic water diffusion in the nervous system-a technical review. NMR Biomed 15:435-455.

Beck AT, Ward CH, Mendelson M, Mock J, Erbaugh J (1961) An inventory for measuring depression. Arch Gen Psychiatry 4:561-571.

Bishop SJ, Duncan J, Lawrence AD (2004) State anxiety modulation of the amygdala response to unattended threat-related stimuli. J Neurosci 24:10364-10368.

Cloninger CR (1986) A unified biosocial theory of personality and its role in the development of anxiety states. Psychiatr Dev 4:167-226.

Costa PT Jr, McCrae RR (1997) Stability and change in personality assessment: the revised NEO Personality Inventory in the year 2000. J Pers Assess 68:86-94.

Cox RW (1996) AFNI: software for analysis and visualization of functional magnetic resonance neuroimages. Comput Biomed Res 29:162-173.

Croxson PL, Johansen-Berg H, Behrens TE, Robson MD, Pinsk MA, Gross CG, Richter W, Richter MC, Kastner S, Rushworth MF (2005) Quantitative investigation of connections of the prefrontal cortex in the human and macaque using probabilistic diffusion tractography. J Neurosci 25:8854-8866.

Dickie EW, Armony JL (2008) Amygdala responses to unattended fearful faces: interaction between sex and trait anxiety. Psychiatry Res 162:51-57. 
Ebeling U, von Cramon D (1992) Topography of the uncinate fascicle and adjacent temporal fiber tracts. Acta Neurochir (Wien) 115:143-148.

Ekman PF, Friesen WV (1976) Pictures of facial affect. Palo Alto: Consulting Psychologists.

Etkin A, Wager TD (2007) Functional neuroimaging of anxiety: a metaanalysis of emotional processing in PTSD, social anxiety disorder, and specific phobia. Am J Psychiatry 164:1476-1488.

Etkin A, Klemenhagen KC, Dudman JT, Rogan MT, Hen R, Kandel ER, Hirsch J (2004) Individual differences in trait anxiety predict the response of the basolateral amygdala to unconsciously processed fearful faces. Neuron 44:1043-1055.

Ferry AT, Ongür D, An X, Price JL (2000) Prefrontal cortical projections to the striatum in macaque monkeys: evidence for an organization related to prefrontal networks. J Comp Neurol 425:447-470.

Fudge JL, Kunishio K, Walsh P, Richard C, Haber SN (2002) Amygdaloid projections to ventromedial striatal subterritories in the primate. Neuroscience 110:257-275.

Ghashghaei HT, Hilgetag CC, Barbas H (2007) Sequence of information processing for emotions based on the anatomic dialogue between prefrontal cortex and amygdala. Neuroimage 34:905-923.

Haas BW, Omura K, Constable RT, Canli T (2007) Emotional conflict and neuroticism: personality-dependent activation in the amygdala and subgenual anterior cingulate. Behav Neurosci 121:249-256.

Hare TA, Tottenham N, Galvan A, Voss HU, Glover GH, Casey BJ (2008) Biological substrates of emotional reactivity and regulation in adolescence during an emotional go-nogo task. Biol Psychiatry 63:927-934.

Highley JR, Walker MA, Esiri MM, Crow TJ, Harrison PJ (2002) Asymmetry of the uncinate fasciculus: a post-mortem study of normal subjects and patients with schizophrenia. Cereb Cortex 12:1218-1224.

Johansen-Berg H, Gutman DA, Behrens TE, Matthews PM, Rushworth MF, Katz E, Lozano AM, Mayberg HS (2008) Anatomical connectivity of the subgenual cingulate region targeted with deep brain stimulation for treatment-resistant depression. Cereb Cortex 18:1374-1383.

Kim H, Somerville LH, Johnstone T, Alexander AL, Whalen PJ (2003) Inverse amygdala and medial prefrontal cortex responses to surprised faces. Neuroreport 14:2317-2322.

Maldjian JA, Laurienti PJ, Kraft RA, Burdette JH (2003) An automated method for neuroanatomic and cytoarchitectonic atlas-based interrogation of fMRI data sets. Neuroimage 19:1233-1239.

Milad MR, Quirk GJ (2002) Neurons in medial prefrontal cortex signal memory for fear extinction. Nature 420:70-74.

Oldfield RC (1971) The assessment and analysis of handedness: the Edinburgh inventory. Neuropsychologia 9:97-113.

Pacheco J, Beevers CG, Benavides C, McGeary J, Stice E, Schnyer DM (2009) Frontal-limbic white matter pathway associations with the serotonin transporter gene promoter region (5-HTTLPR) polymorphism. J Neurosci 29:6229-6233.

Pezawas L, Meyer-Lindenberg A, Drabant EM, Verchinski BA, Munoz KE, Kolachana BS, Egan MF, Mattay VS, Hariri AR, Weinberger DR (2005) 5-HTTLPR polymorphism impacts human cingulate-amygdala interactions: a genetic susceptibility mechanism for depression. Nat Neurosci 8:828-834.

Rauch SL, Whalen PJ, Shin LM, McInerney SC, Macklin ML, Lasko NB, Orr SP, Pitman RK (2000) Exaggerated amygdala response to masked facial stimuli in posttraumatic stress disorder: a functional MRI study. Biol Psychiatry 47:769-776.

Rueckert D, Sonoda LI, Hayes C, Hill DL, Leach MO, Hawkes DJ (1999) Nonrigid registration using free-form deformations: application to breast MR images. IEEE Trans Med Imaging 18:712-721.

Selden NR, Gitelman DR, Salamon-Murayama N, Parrish TB, Mesulam MM (1998) Trajectories of cholinergic pathways within the cerebral hemispheres of the human brain. Brain 121:2249-2257.

Shin LM, Wright CI, Cannistraro PA, Wedig MM, McMullin K, Martis B, Macklin ML, Lasko NB, Cavanagh SR, Krangel TS, Orr SP, Pitman RK, Whalen PJ, Rauch SL (2005) A functional magnetic resonance imaging study of amygdala and medial prefrontal cortex responses to overtly presented fearful faces in posttraumatic stress disorder. Arch Gen Psychiatry 62:273-281.

Smith SM, Jenkinson M, Woolrich MW, Beckmann CF, Behrens TE, Johansen-Berg H, Bannister PR, De Luca M, Drobnjak I, Flitney DE, Niazy RK, Saunders J, Vickers J, Zhang Y, De Stefano N, Brady JM, Matthews PM (2004) Advances in functional and structural MR image analysis and implementation as FSL. Neuroimage 23 [Suppl 1]:S208-S219.

Spielberger CD, Gorsuch RL, Lushene RE (1988) STAI: manual for the State Trait Anxiety Inventory, Ed 3. Palo Alto, CA: Consulting Psychologists.

Tuch DS, Salat DH, Wisco JJ, Zaleta AK, Hevelone ND, Rosas HD (2005) Choice reaction time performance correlates with diffusion anisotropy in white matter pathways supporting visuospatial attention. Proc Natl Acad Sci U S A 102:12212-12217.

Urry HL, van Reekum CM, Johnstone T, Kalin NH, Thurow ME, Schaefer HS, Jackson CA, Frye CJ, Greischar LL, Alexander AL, Davidson RJ (2006) Amygdala and ventromedial prefrontal cortex are inversely coupled during regulation of negative affect and predict the diurnal pattern of cortisol secretion among older adults. J Neurosci 26:4415-4425.

Wang F, Kalmar JH, He Y, Jackowski M, Chepenik LG, Edmiston EE, Tie K, Gong G, Shah MP, Jones M, Uderman J, Constable RT, Blumberg HP (2009) Functional and structural connectivity between the perigenual anterior cingulate and amygdala in bipolar disorder. Biol Psychiatry 66: $516-521$. 